\title{
Biomarkers of alcohol misuse: recent advances and future prospects
}

\author{
Iwona Jastrzębska ${ }^{1}$, Agnieszka Zwolak ${ }^{1}$, Michat Szczyrek ${ }^{1}$, Agnieszka Wawryniuk ${ }^{1}$, \\ Barbara Skrzydło-Radomańska², Jadwiga Daniluk ${ }^{1}$ \\ ${ }^{1}$ Chair of Internal Medicine and Department of Internal Medicine in Nursing, Medical University of Lublin, Lublin, Poland \\ ${ }^{2}$ Chair and Department of Gastroenterology with Endoscopic Unit, Medical University of Lublin, Lublin, Poland
}

Gastroenterology Rev 2016; 11 (2): 78-89

DOI: $10.5114 /$ pg.2016.60252

Key words: alcohol misuse, alcohol abuse, alcohol biomarkers.

Address for correspondence: Iwona Jastrzębska MD, PhD, Chair of Internal Medicine and Department of Internal Medicine in Nursing, Medical University of Lublin, 8 Jaczewskiego St, 20-954 Lublin, Poland, phone/fax: +48 8174258 25, e-mail: ivjastrzebska@wp.pl

\begin{abstract}
Alcohol abuse and dependence are highly prevalent in many cultures and contribute considerably to the global burden of health and social issues. The current inability to accurately characterise long-term drinking behaviours is a major obstacle to alcoholism diagnosis and treatment. Therefore, it is of great importance to develop objective diagnostic tools to discern subjects with excessive alcohol use and alcoholism or to confirm abstinence. Research over past years has revealed several biochemical compounds with considerable potential for accurate reflection of alcohol intake. This review will address the issue of alcohol biomarker definition, the types of molecules used as so-called traditional biomarkers, and the compounds that can serve as novel biomarker candidates or components of biomarker panels.
\end{abstract}

\section{Introduction}

The use of alcoholic beverages is probably the most ancient social habit worldwide, it is highly prevalent in many cultures and contributes considerably to the global burden of health and social issues. Chronic and acute alcohol intoxication has been linked to a multitude of diseases, including cancers, cardiovascular diseases, liver cirrhosis, neuropsychiatric disorders, injuries, and foetal alcohol syndrome, as well as social problems, such as suicides, homicides, road and industrial accidents, and many criminal offences [1, 2].

The inability to properly assess alcohol drinking behaviours (see Table I for the main patterns of alcohol misuse) presents a significant barrier to the diagnosis and treatment of hazardous alcohol use. It is important to emphasise that there are no pathognomonic clinical signs or symptoms of alcoholism. Medical complaints and clinical presentation of individuals consuming excessive amounts of alcohol depend on when in the course of the condition they seek medical attention. One approach for detecting hazardous drinking is to ask a simple screening question, followed by a more focused self-report questionnaire if required. There are a number of relatively easy questionnaires available for quantifying alcohol intake, including CAGE (named after the key questions asked in the questionnaires), the Michigan Alcoholism Screening Test (MAST), the Alcohol Use Disorders Identification Test (AUDIT), and its shortened version (AUDIT-C) [3-5]. Although meticulous efforts have been made to construct these interview formats there are serious limitations associated with these approaches. A number of patients fail to admit to their true alcohol consumption, particularly when they are forced to deny or minimise the magnitude of drinking behaviour in order to mitigate personal, professional, or legal ramifications of alcohol abuse, or in the context of altered mental states or mental illnesses [5]. The issue coupled with physician discomfort, inadequate training, or judgmental attitudes makes the diagnostic process even more complex.

Therefore, it is of great importance to have objective diagnostic tools to discern subjects with excessive alcohol use and alcoholism or to confirm abstinence. This review will address the issue of alcohol biomarker definition, the types of molecules used as so-called traditional biomarkers, and the compounds that can 
Table I. The main patterns of alcohol misuse $[8,9]$

\begin{tabular}{|c|c|c|}
\hline Pattern of alcohol misuse & $\begin{array}{l}\text { Level of alcohol consumption/ } \\
\text { number of units* }\end{array}$ & Risk/problems \\
\hline Social drinking & $\begin{array}{l}\text { Usually drinking not more than } \\
2-3 \text { units of alcohol/day }\end{array}$ & $\begin{array}{l}\text { The term refers to substance use that poses low risk of future } \\
\text { harmful consequences for the user }\end{array}$ \\
\hline Hazardous drinking & $\begin{array}{l}\text { Women who drink more than } \\
\text { 1-2 units of alcohol/day and men } \\
\text { who drink more than 3-4 units/day }\end{array}$ & $\begin{array}{l}\text { The term refers to substance use that increases the risk of } \\
\text { harmful consequences for the user. The pattern is of public health } \\
\text { significance despite the absence of any current disorder in the } \\
\text { individual user }\end{array}$ \\
\hline $\begin{array}{l}\text { Binge drinking (also called } \\
\text { single occasion drinking) }\end{array}$ & $\begin{array}{l}\text { Occasionally (i.e. in one drinking } \\
\text { session) drinking more than } \\
5 \text { units of alcohol/day }\end{array}$ & $\begin{array}{l}\text { The term refers to an episode of heavy drinking over a short period } \\
\text { of time (e.g. over the course of an evening or over an hour or two), } \\
\text { or drinking to intoxication or to drunkenness }\end{array}$ \\
\hline $\begin{array}{l}\text { Heavy drinking (also called } \\
\text { harmful drinking or alcohol } \\
\text { abuse) }\end{array}$ & $\begin{array}{l}\text { Drinking regularly more than } 6 \text { units } \\
\text { of alcohol/day }\end{array}$ & $\begin{array}{l}\text { The term refers to drinkers already experiencing or showing evidence } \\
\text { of health harms (physical or mental), but not showing evidence of } \\
\text { alcohol dependence. If immediate harm appears, they are called } \\
\text { problem drinkers }\end{array}$ \\
\hline Dependent drinking & Drinking alcohol chronically & $\begin{array}{l}\text { The most severe stage of drinking, with physical and psychological } \\
\text { dependence. At least three of the following criteria are met: } \\
\text { tolerance, withdrawal symptoms after cessation of drinking, } \\
\text { impaired control, preoccupation with acquisition and/or use, } \\
\text { persistent desire or unsuccessful efforts to quit, sustains social, } \\
\text { occupational or recreational disability, and use continues despite } \\
\text { adverse consequences }\end{array}$ \\
\hline
\end{tabular}

serve as novel biomarker candidates or components of biomarker panels.

\section{Biomarkers of alcohol consumption}

In accordance with the definition established by the US National Institute of Health, a biomarker is "a characteristic that is objectively measured and evaluated as an indicator of normal biological processes, pathogenic processes, or pharmacologic responses to a therapeutic intervention" [6]. Thus, in the context of alcohol abuse, a biomarker should be an accurate indicator of an individual's alcohol drinking pattern, or any genetic predisposition toward alcohol abuse and alcoholism. These two kinds of alcohol biomarkers are described as state markers (i.e. biochemical measures that allow evaluation of a patient's history of alcohol consumption) and trait markers (i.e. biochemical tools that reveal a person's inherited risk of developing alcoholism due to chronic exposure), respectively [7]. Importantly, the potential utility of a biomarker is strictly defined by its diagnostic power. To qualify an alcohol-related biomarker as useful; it should meet several criteria, of which sensitivity (the diagnostic method has to be accurate for most if not all drinking subjects) and specificity (the diagnostic method has to be linked to alcohol consumption but not to other conditions or problems) are of utmost importance. From practical point of view, the test used to measure the biomarker should also be precise and accurate $[6,7]$.
State markers of excessive alcohol intake can be grouped into two types due to pathophysiological matters: indicators of alcohol consumption (acute as well as chronic) and indicators of alcohol induced organ damage. Biochemical measures traditionally linked with hazardous drinking are $\gamma$-glutamyl transferase (GGT), aspartate aminotransferase (AST), alanine aminotransferase (ALT), mean cell volume (MCV), and carbohydrate-deficient transferrin (CDT) [7-9]. However, in a broad sense, their utility as diagnostic tools for alcohol abuse is greatly hampered due to variable results in different populations and low sensitivity and specificity (Tables II and III). Nevertheless, it is worth emphasising that CTD is the first test approved by the U.S. Food and Drug Administration for the monitoring of alcohol consumption over an extended period of time $[8,9]$.

\section{Novel state markers}

Research in recent years has revealed several biochemical markers with considerable potential for more accurate reflection of excessive alcohol intake than traditional markers. These markers may be related to compounds produced when the body metabolises alcohol (so-called direct markers) or may reflect changes in other molecules, cells, or tissues that result from chronic or acute alcohol exposure (so-called indirect markers). Most of them have been developed in a research context and are still awaiting validation and possible introduction into commercial settings [7-10]. 


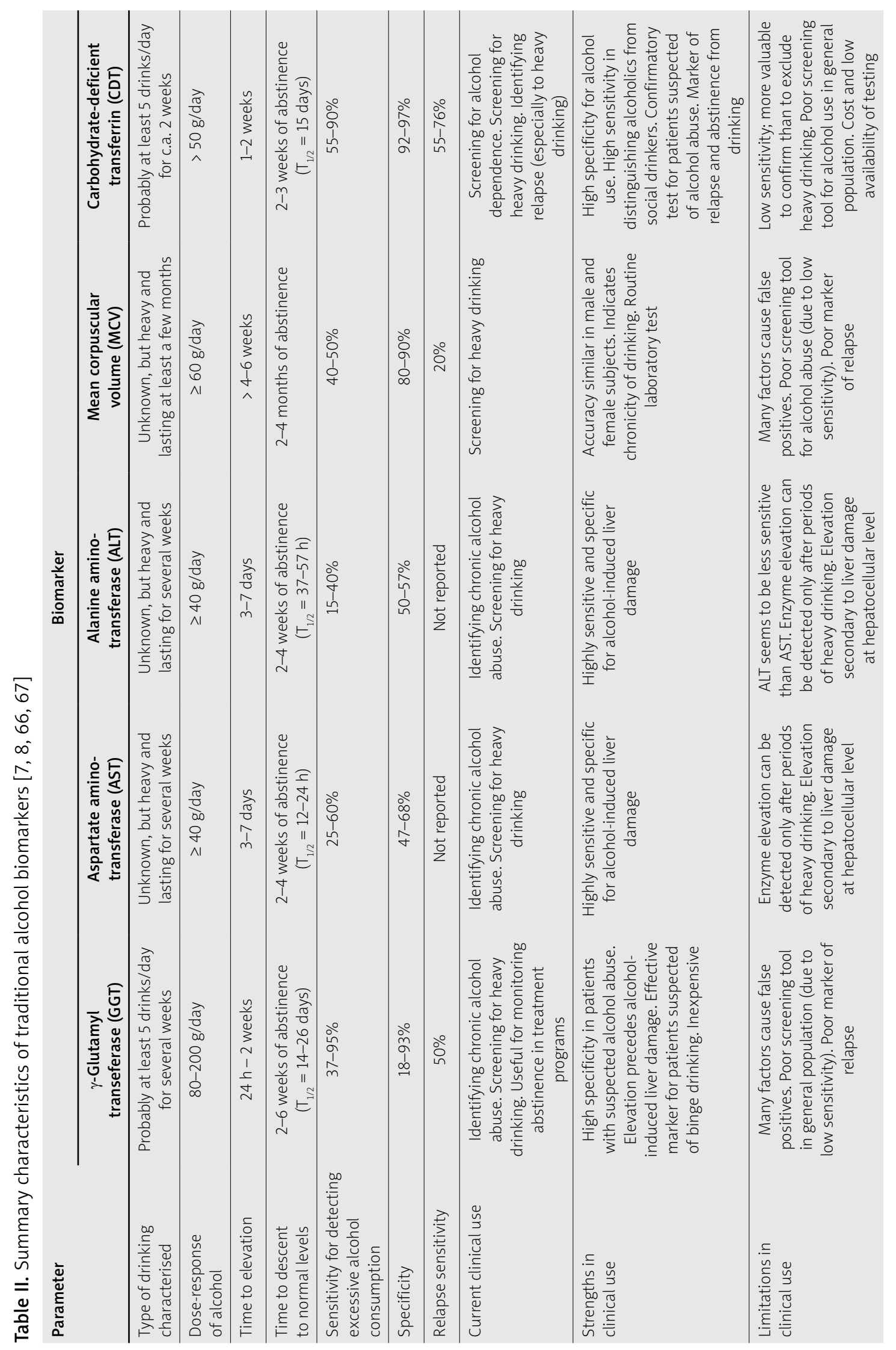




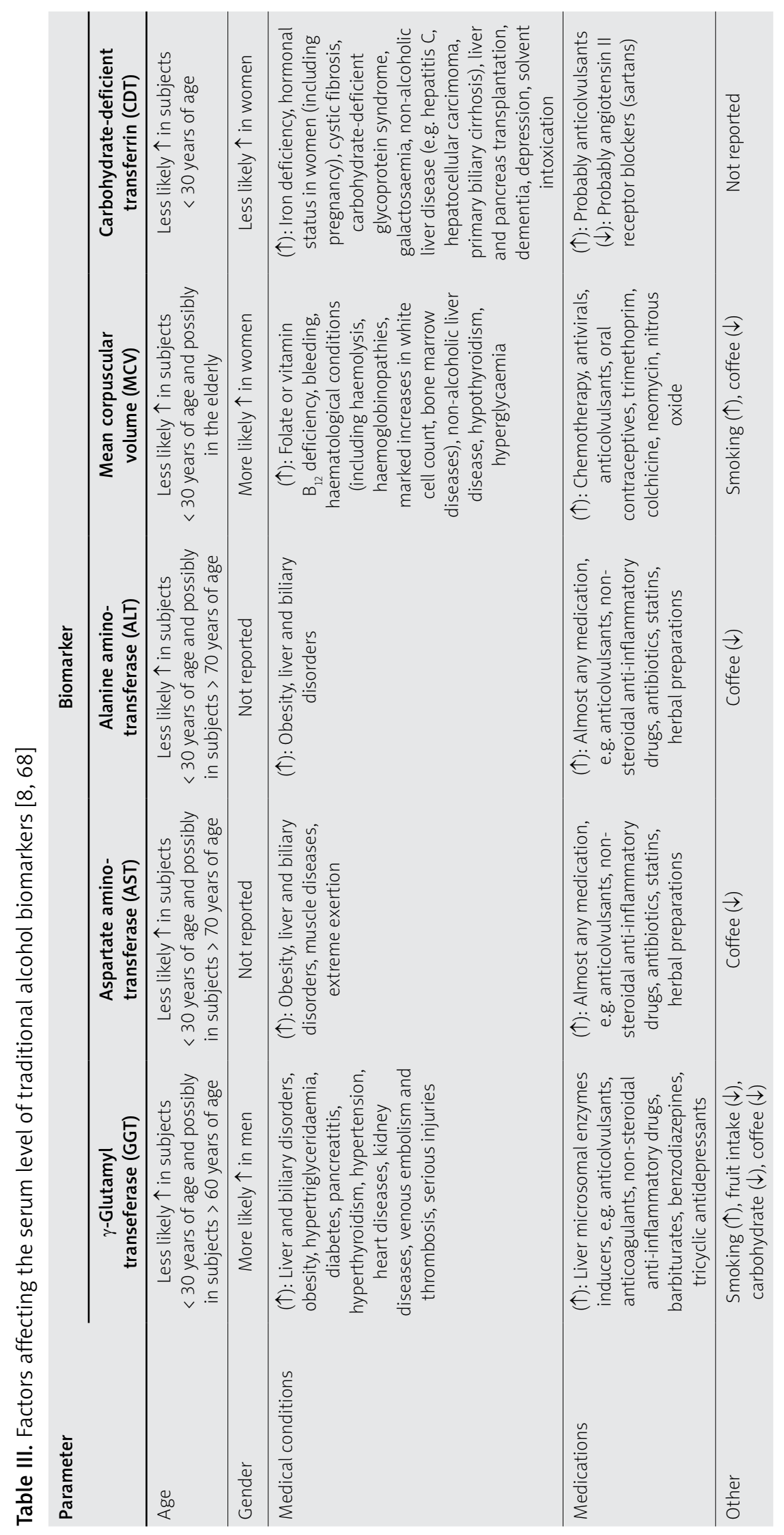




\section{Ethyl glucuronide, ethyl sulphate}

Ethyl glucuronide (EtG) and ethyl sulphate (EtS) are direct conjugated metabolites of ethanol formed in low amounts primarily in the endoplasmic reticulum of liver, and the reactions are catalysed by uridine diphosphate-glucuronosyltransferase and sulphotransferase, respectively [7, 9, 11]. Compared with ethanol testing, EtG and EtS are superior markers of recent alcohol intake due to a longer detection window. Namely, EtG is present in blood for up to $36 \mathrm{~h}$ (c.a. $8 \mathrm{~h}$ following complete elimination of ethanol) and in urine for 3-5 days after heavy alcohol consumption [7, 9], whereas EtS is detectable in urine c.a. 16-27 $\mathrm{h}$ longer than ethanol [12]. When people test positive for EtG, it is likely that they have consumed alcohol recently, even if there is no ethanol left in their bodies. This makes EtG especially useful for detecting drinking relapses, particularly in alcoholism treatment programs. In addition to blood and urine, EtG can be also detected in other body fluids, hair, and body tissues [7, 9, 13]. A few studies have demonstrated that EtG measurements in hair have a relatively high specificity and sensitivity in the detection of alcohol abuse: $80-95 \%$ and $70-90 \%$, respectively $[14,15]$.

Importantly, EtG/EtS results should be interpreted in the context of all available clinical and behavioural information. It has been reported that incidental exposure to alcohol in daily use products (i.e. hand sanitisers, mouthwash) may result in detectable levels of EtG and/or EtS. In addition, upper respiratory infections as well as $\beta$-glucuronidase hydrolysis may lower levels of EtG, but do not seem to affect EtS [16, 17]. Furthermore, EtG in hair is vulnerable to cosmetic treatments [18]. Another weakness of EtG as a biomarker of alcohol misuse is a rather sophisticated method required for an accurate reading of EtG from urine, and so far, attempts to produce a measure for urine-based EtG using simpler techniques or to measure EtG in other body fluids or hair have yielded less than satisfactory results $[7,9,11]$.

\section{Acetaldehyde, acetaldehyde adducts, and anti-adduct antibodies}

Acetaldehyde is a first product of oxidative metabolism of ethanol. The circulating compound exists on its own, but has also been demonstrated to react with various proteins (including haemoglobin, albumin, and other serum proteins, CYP450 2E1, or red blood cell membrane proteins), resulting in acetaldehyde-protein adduct formation [7, 10, 19]. Following alcohol intake, concentration of free acetaldehyde is highly variable with a life-time of c.a. $3 \mathrm{~h}$, but some acetaldehyde-protein adducts may be detected up to 3 weeks after alcohol consumption, and haemoglobin-bound acetaldehyde (HAA) accumulates in red blood cells over their 120-day average life span $[7,10,20]$. A single highdose of alcohol $(2 \mathrm{~g} / \mathrm{kg})$ increases blood HAA when the conventional markers, such as MCV or GGT, show no change $[9,10]$. Approaches aimed at detecting both free and bound acetaldehyde in blood have been developed. So-called whole blood-associated acetaldehyde assay (WBAA) has great potential as a highly specific, precise, and extremely sensitive tool to test for heavy alcohol consumption and to monitor people in alcoholism treatment programs. The ability of the WBAA or HAA assays to measure alcohol consumption patterns over time make them unique among the biomarkers of alcohol misuse [7].

Alternatively, circulating antibodies against acetaldehyde adducts may be measured as biomarkers of alcohol intake. Binding with human proteins, acetaldehyde gives rise to a molecular adduct having acetaldehyde as hapten, and therefore forming neo-antigen that can induce antibodies toward themselves [9, 21]. The increased reactivity of IgA with acetaldehyde-modified serum proteins has been reported in heavy drinkers and alcohol-dependent persons, but not in social drinkers. Furthermore, an increased ratio of IgA/IgG is highly indicative of alcoholic liver disease [9]. However, the sensitivity and specificity of the circulating anti-adduct antibodies are $65-73 \%$ and $88-94 \%$, respectively [22]. Interestingly, a single high-dose of alcohol $(2 \mathrm{~g} / \mathrm{kg})$ has been shown to increase the level of salivary IgA. As saliva is an easily and non-invasively obtained material, salivary anti-acetaldehyde adducts IgAs seem to show promise in binge drinking detection [23].

\section{Fatty acid ethyl esters}

Fatty acid ethyl esters (FAEEs) represent products of non-oxidative ethanol metabolism. The compounds are formed by conjugation between fatty acyl chains (such as oleic acid, steric acid, and palmitic acid) and ethanol. The reaction is most often catalysed by enzymes (such as FAEE synthase, microsomal acyl-CoA:ethanol o-acyltransferase, carboxylesterase, lipoprotein lipase, cholesterol esterase, or triglyceride lipase), but FAEEs can also be formed spontaneously [7]. These alcohol metabolites are present throughout the body, including pancreas, liver, heart, brain, white blood cells, adipose tissue, hair, blood, and meconium, and they accumulate preferentially in adipose tissue and hair [24, 25].

Along with acetaldehyde, FAEE formation is an important pathway of ethanol disposition. Fatty acid ethyl esters measured in liver and adipose tissue have been used as a postmortem marker of alcohol consumption. Human and animal studies have demonstrated that when measured in adipose tissue, FAEEs may be useful as an alcohol consumption marker for up to $12 \mathrm{~h}$ af- 
ter death; whereas when measured in liver tissue, they may be useful for up to $24 \mathrm{~h}$ after alcohol consumption [26]. Such a tool is needed because traditional measures, e.g. blood alcohol levels, can be artificially high as a result of postmortem alcohol formation. Furthermore, FAEEs measured in hair look very promising as a marker providing long-term insight into drinking behaviour, as there is no possibility to flush them out of hair and they build up over a long period of chronic drinking. Interestingly, FAEEs have been found to accumulate within the proximal $5-10 \mathrm{~cm}$ of hair, and then to decrease to a plateau afterwards, irrespective of alcohol dose. Therefore, through segmental analysis of the hair, it seems to be possible to confirm a current period of prolonged abstinence if FAEE levels consistent with heavy alcohol exposure are not found in newly grown segments [25]. The FAEE hair test that measures cumulative levels of four separate molecules (ethyl myristate, palmitate, oleate, and stearate) seems to be a sensitive and specific marker for chronic excessive alcohol use in adults, distinguishing social drinkers from heavy or alcohol-dependent drinkers $[24,27]$. With the appropriate cut-off level ( $0.5 \mathrm{ng}$ of cumulative FAEEs per mg hair) it has been found to be $90 \%$ sensitive and specific in the detection of heavy alcohol use. Hair FAEE levels between 0.2 and $0.5 \mathrm{ng} / \mathrm{mg}$ have been found to be indicative of social use, typically excluding strict abstainers, whereas levels in excess of $1.0 \mathrm{ng} / \mathrm{mg}$ are nearly $100 \%$ specific to heavy alcohol use, but offer lower sensitivity $(\sim 75 \%)[27,28]$. One should remember that FAEEs in hair can be affected by cosmetic treatments and hair care, although preliminary studies seem to indicate that these effects are not likely to be of clinical significance [29]. Additionally, FAEEs through their measurement in meconium may prove to have unique value as a reliable test providing evidence of prenatal exposure to alcohol in newborn infants and therefore for drinking during pregnancy [30, 31].

\section{Phosphatidylethanol}

Phosphatidylethanol (PEth) represents an abnormal cellular membrane phospholipid formed only in the presence of ethanol. Though the reaction catalysed by phospholipase D has been detected in the whole body, for the purpose of serving as an alcohol consumption biomarker PEth is sampled in the blood cells, where it can be readily accessed and measured [32]. In vitro studies have demonstrated that the quantity of PEth produced in human red blood cells is directly proportional to the ethanol concentration and the exposure time, and there is no correlation between the rate of PEth formation and haematological indexes (i.e. red blood cell count, mean corpuscular volume, haemato- crit). Furthermore, the absence of PEth enzymatic degradation activity in human erythrocytes results in its accumulation in cellular membranes, which suggests a potential use of the compound for the measurement of prolonged as well as binge alcohol consumption. Importantly, PEth is considered to be less sensitive than EtG or EtS to small amounts of ethanol and does not detect single drink episodes [32]. The threshold of total ethanol intake resulting in a positive PEth assay was estimated at c.a. $1000 \mathrm{~g}$ in 3 weeks, with a daily consumption of at least $50 \mathrm{~g}$ [33].

Since the formation of PEth is specifically dependent on ethanol, the diagnostic specificity of PEth as an alcohol biomarker is theoretically $100 \%$. Remarkably, its sensitivity has been found to reach high values, between 94.5 and 100\% [34, 35]. Differing from the traditional indirect biomarkers used for diagnosing a chronic excessive drinking behaviour (i.e., MCV, AST, ALT, GGT, and CDT), blood PEth concentration seems not to be influenced by age, gender, other ingested substances, or non alcohol-associated diseases, such as hypertension, and kidney and/or liver diseases. In contrast to EtG or EtS, PEth is considered to be insensitive to incidental ethanol exposures, such as mouthwash and antibacterial hand cleansers $[33,34]$. The utility of PEth for the diagnosis of alcohol abuse is determined by the shortterm nature of this marker, its mean half-life in blood of alcoholics is approximately 4 days, with a range of 3-5.3 days. In clinical studies, the compound was detectable in blood of chronic heavy drinkers up to 28 days after sobriety [33]. Despite such high performance, the existing methods for detecting PEth are still too challenging for routine clinical usage, although they are capable of effectively measuring single molecular species of PEth in blood in the nanomolar range.

\section{$\beta$-Hexosaminidase}

$\beta$-Hexosaminidase $(\beta$-HEX) is a lysosomal exoglycosidase present in most cell types, and it is normally involved in the metabolism of carbohydrates and gangliosides in the hepatocytes, particularly in release of $\mathrm{N}$-acetylhexosamines from the non-reducing end of oligosaccharide chains of glycoconjugates [9, 10, 36]. Heavy alcohol consumption, i.e. more than $60 \mathrm{~g}$ per day for at least 10 consecutive days, results in marked changes of the enzyme activity in the body fluids. One proposed mechanism is lysosomes damage and subsequent leakage of the enzyme from lysosomes and cells into body fluids [36]. The diagnostic sensitivity of increased activity of the serum $\beta$-HEX B isoenzyme and urine total $\beta$-HEX has been reported to achieve $69-94 \%$ and $81-85 \%$, respectively. Furthermore, in alcohol-dependent persons, the enzyme levels fall rapidly to nor- 
mal following abstinence (7-10 days, $\mathrm{T}_{1 / 2}=6.5$ days). $[10,37]$. However, $\beta$-HEX serum, urine, or saliva levels may also increase following isolated consumption of ca. $2 \mathrm{~g} / \mathrm{kg}$ bw of alcohol (so-called "binge drinking") [38]. Despite relatively high specificity (84-98\%), subjects with liver disorders (such as cholestasis and cirrhosis), hypertension, diabetes mellitus, cerebral or myocardial infarction, thyrotoxicosis, pregnancy, or after oral contraceptive pills may present with a false-positive elevation of the enzyme activity $[10,37]$. The distinct advantage of $\beta$-HEX as a potential marker of prolonged alcohol abuse is that it is a standard and inexpensive technique of detection.

\section{Plasma Sialic Acid Index of Apolipoprotein J}

The term "Plasma Sialic Acid Index of Apolipoprotein J" (SIJ) expresses the ratio of moles of sialic acid per mole of apolipoprotein J (Apo J). Apolipoprotein J (or clusterin) is a multifunctional $\mathrm{N}$-glycoprotein found in high-density lipoprotein (HDL) complexes, which has been implicated in a plethora of physiological and pathological processes. Among others, it is considered to be implicated in lipid transfer between lipoproteins, especially cholesterol. The glycoprotein is highly sialylated, i.e. human Apo J was demonstrated to contain 28 moles of sialic acid residues per mole of Apo J, compared with 4-6 moles of sialic acid per mole of transferrin [9]. This is highly significant and may make it easier to measure changes in sialic acid content caused by alcohol consumption. As with the molecule transferrin, long-term ethanol intoxication decreases sialylation of plasma Apo J, mainly by increasing the activity of sialidase and decreasing cellular glycosyltransferases (i.e. mannosyltransferase, galactosyltransferase, $\mathrm{N}$-acetyl-glucosaminyltransferase, sialyltransferase) [9, 39]. Consequently, SIJ is decreased in alcoholics (on average by $50-57 \%$, with specificity $\sim 100 \%$ ) and its levels progressively return to normal range over a period of several weeks of abstinence $\left(T_{1 / 2}=4-5\right.$ weeks) $[35,40]$. Furthermore, plasma SIJ correlates with relapse in alcohol-dependent subjects ( $\sim 90 \%$ sensitivity) [9].

More studies are needed, but preliminary findings show promise for SIJ as a highly specific marker for alcohol misuse. However, widespread use in any clinical laboratory setting will require simplification of the method of measuring sialic acid in plasma Apo J. Although the methods used in most studies have been relatively straightforward and cost-effective, they are highly sophisticated and time-consuming, and they can be carried out only in specialised laboratories at the present time.

\section{Total serum sialic acid}

In humans, serum sialic acids are mostly attached to carbohydrate chains of glycoproteins, e.g. transferrin and Apo J, and glycolipids. Apart from these two fractions of sialic acid, serum total sialic acid (TSA) also comprises a minor fraction of serum free sialic acid (FSA) generated by desialylation of glycoproteins. The serum levels of TSA and FSA during excessive alcohol consumption seem to depend on the changes in the most sialylated glycoproteins [41].

Literature data have demonstrated that TSA concentration has clear potential as a marker for excessive alcohol consumption. Compared with social drinkers, both male and female alcoholics have elevated amounts of TSA in the serum, saliva, and urine, although the exact mechanisms that generate this increase still remain a matter of speculation $[9,41,42]$. The diagnostic value of TSA as an alcohol abuse biomarker showed 48-58\% sensitivity and 64-96\% specificity [9, 41]. Unfortunately, various diseases and states, such as cancer, diabetes, renal diseases, cardiovascular disease, or pregnancy, may increase serum TSA concentration, thus decreasing its specificity [43]. However, in a research context the TSA levels did not differ between subjects with elevated and normal liver enzymes activity, in contrast to a fraction of lipid-bound sialic acid [42]. Despite the lack of specificity, TSA can be recognised as a good test of alcohol abuse, independent of the presence of hepatocellular injury. Because TSA levels take longer than either CDT or GGT to decrease during periods of abstinence, the TSA test might not be useful for treatment programs assessing patients for relapse.

Interestingly, preliminary studies have demonstrated elevated concentrations of FSA in the sera of alcoholics. The diagnostic accuracy reached the value of $85-94 \%$, although the sensitivity was low ( 40\%). Compared with traditional markers of alcohol abuse, the clinical usefulness of FSA is markedly lower than that of CDT and GGT, even though the specificity and positive predictive value of those tests were similar. At present, the clinical significance of FSA is limited to inherited diseases, including sialidosis, Salla disease, infantile sialic acid storage disease, and neuraminidase deficiency [41, 44].

\section{Cholesteryl ester transfer protein}

Cholesteryl ester transfer protein (CETP) is a hydrophobic glycoprotein synthesised by hepatocytes and circulating in plasma bound mainly to HDL particles. It promotes the redistribution of cholesteryl esters, triacylglycerols, and phospholipids between lipoproteins [9]. Research data have demonstrated that alcohol consumption markedly reduces both the plasma concentration and activity of CETP. As a consequence 
the direction of cholesteryl esters transfer is reversed, which leads to an increase in the plasma HDL cholesterol concentration (a common laboratory abnormality in alcohol-dependent persons) $[9,37]$. The possible clinical usefulness of plasma CETP is considered to be comparable with conventional alcohol markers, such as MCV, GGT, AST, and ALT. Its specificity is, however, limited due to some factors affecting plasma levels (e.g. various diseases, differences in diet, drugs) [9]. Another disadvantage of CETP as a marker of alcohol misuse is its complicated and laborious method of determination.

\section{5-Hydroxytryptophol,}

\section{5-hydroxyindole-3-acetic acid}

5-hydroxytryptophol (5-HTOL) comprises one of the minor metabolites of the neurotransmitter serotonin, and it is a normal constituent of urine. Alcohol and its primary oxidative metabolite, acetaldehyde, affect the metabolism of serotonin so that 5-HTOL concentration increases dramatically following alcohol consumption. Elevated 5-HTOL can be detected in urine for 5-15 h (depending on dose) after alcohol intake, compared to standard measurements, which can detect ethanol in the urine for a little over an hour for each drink consumed [45]. Preliminary work indicates that testing for 5-HTOL in urine is both sensitive and specific for detecting recent heavy alcohol consumption and may prove to be especially useful in forensic toxicology. Furthermore, there is a possibility to use the test to monitor subjects involved in treatment maintenance programs (excluding persons treated with the anti-drinking medication disulfiram, which can also lead to increases in 5-HTOL levels) [45].

The ratio of $5-\mathrm{HTOL}$ to another serotonin metabolite, 5-hydroxyindole-3-acetic acid (5-HIAA), is considered to be alternating marker that can be used to verify the presence of ethanol in the body [37]. The 5-HTOL: 5 -HIAA ratio was found to have $100 \%$ sensitivity up to $4 \mathrm{~h}$ after a moderate dose of ethanol, but its reliability decreases fairly rapidly after $7 \mathrm{~h}$ [46]. The short time frame and the sophisticated method of determination currently used limit the diagnostic utility of these markers for assessing the history of ethanol abuse, and hamper their translation into routine clinical practice [37].

\section{Salsolinol}

Salsolinol, formed in brain and other tissue following alcohol intake, is a potential neurotoxin suspected to contribute to alcohol abuse. Chemically it is a biologically active alkaloid with morphine-like effects [47]. The compound primarily constitutes a non-enzymatic condensation product of the neurotransmitter dopamine and the ethanol oxidation product acetaldehyde, although it may also be the effect of the enzymatic reaction between alcohol and pyruvate (a glucose metabolite that is used by cells for energy) [7]. The usefulness of salsolinol as a potential marker for chronic alcohol consumption depends considerably on the method of determination and especially on the tissue in which it is detected [48]. Following acute alcohol consumption, the total urinary salsolinol output and the plasma concentration of salsolinol have been demonstrated to change in different ways. Some alcohol-ingesting subjects showed a significant increase of both urine and plasma concentrations, whereas others presented with decreased or unchanged salsolinol levels. Furthermore, compared with non-alcoholics, alcoholics who have been abstinent for as little as 1 week have decreased salsolinol levels in one type of white blood cell (namely, lymphocytes) [49]. Studies of salsolinol levels in the brain, in contrast, found no difference in salsolinol levels between alcoholics and non-alcoholics [48]. A few studies have reported that salsolinol from dietary sources (e.g. bananas) is a major contributor to its plasma levels [50].

Poor assay specificity and possible artefact formation of the alkaloids during work-up and storage have been suggested to be responsible for controversial reports on the detection of the compound in mammalian tissues and fluids after alcohol intake. Moreover, the analytical technique for determining the compound in human urine, plasma, brain, or cerebrospinal fluid requires sophisticated and expensive special equipment and is therefore not suitable for routine analysis and is unlikely to become clinically valuable [48]. More experimental work is necessary to determine whether alcohol really has an influence on the biosynthesis of salsolinol and if it may be a sufficient clinical marker to distinguish between alcoholics and non-alcoholics.

\section{Dolichol}

Dolichol, a homologous group of $\alpha$-saturated longchain polyisoprenoid alcohols, is synthesised from acetate and accumulates in tissues during ageing. Being a glycosyl carrier, the compound is involved in the translational modification of proteins to $\mathrm{N}$-linked glycoproteins. Its function, however, is easily influenced by free radicals, e.g. those generated due to alcohol consumption $[9,10]$.

Both dolichol and ethanol are substrates for a single enzyme, alcohol dehydrogenase, and due to this substrate competition, elevated dolichol levels in the blood and urine have been suggested as markers of alcohol abuse [48]. Importantly, human studies have demonstrated that moderate alcohol consumption (60 g/day) did not affect urinary dolichol levels. Increased urinary dolichol levels have been reported in 
chronic alcoholics and in newborns of alcoholic mothers, and as related to urinary creatinine they were 2.5-4 times higher than that of non-alcoholic social drinkers. The half-life of urinary dolichol is c.a. 3 days, whereas in serum it exceeds 7 days $[37,51,52]$. In alcoholic patients elevated urinary dolichol levels returned to normal by the fifth day of abstinence [51]. Despite the fact that the urinary dolichol test showed high specificity (96\%), its sensitivity is moderate $(68 \%)$ or even low (9-19\%) [37].

\section{Circulating cytokines}

Cytokines are a class of multifunctional proteins implicated in cellular communication and activation. Being involved in processes such as inflammation, cell death, cell proliferation, cell migration, and healing mechanisms, they are critical to the development and functioning of both innate and adaptive immune response [53].

Both acute and long-term alcohol consumption have been demonstrated to influence considerably inflammatory cell and adaptive immune responses and directly suppress a wide range of immune responses. Among other mechanisms, alcohol is known to alter cytokine levels in a variety of tissues including plasma, liver, lung, and brain [53-56]. As the measurement of serum levels of many cytokines has recently become more available in clinical practice, it is possible for circulating cytokines to contribute to diagnostic tools for alcohol abuse. The most promising candidates comprise tumour necrosis factor- $\alpha$ (TNF- $\alpha$ ), interleukin (IL)- $1 \alpha, \mathrm{IL}-1 \beta, \mathrm{IL}-6, \mathrm{IL}-8$, IL-12, and monocyte chemoattractant protein-1 (MCP-1) [53]. Serum levels of TNF- $\alpha$ have been shown to be higher in alcoholics than in the general population, regardless of alcohol consumption level [57]. Circulating TNF- $\alpha, I L-1$, and IL- 6 are found to be elevated in both acute and chronic alcohol-induced liver disease. Furthermore, chronic alcohol consumption without associated liver disease has also been linked with significantly increased production of TNF- $\alpha, \mathrm{IL}-1 \beta, \mathrm{IL}-6$, and IL-12 [53]. On the other hand, subjects with alcohol liver cirrhosis, who were actively drinking, showed abnormally low levels of inflammatory cytokines. Interestingly, no significant alterations in cytokine levels were observed in patients with alcohol liver cirrhosis, who were in alcohol abstinence [58].

Although a growing body of evidence suggests the potential use of circulating cytokines as an indicator of alcohol intake, given their broad biological role, it is unlikely that they will be used as standalone alcohol biomarkers. Similarly, the role of other factors in cytokine release associated with alcoholism, including nutrition, age, gender, method of analysis, and co-morbid drug use, still remains to be elucidated.

\section{Proteomic techniques in the alcohol misuse field}

Proteomics is defined as the analysis of many or all of the proteins in a given sample. The central premise of such analysis is the comprehensive characterisation of the proteins in a cell, tissue, or organ that will provide insights into the status of the system. Based on the methodological considerations, proteomics addresses the physical arrangement of amino acids into a protein (structural proteomics), the actual physiologic activity of proteins (functional proteomics), and the patterns of protein expression and modification in health and disease (expression proteomics). One of the aims of proteomics is to identify biomarkers of disease [59].

Literature data directly indicate that proteomic techniques may constitute powerful tools in the discovery, characterisation, and validation of the complex new biomarker panels for alcohol misuse [60]. The instrumentation and the computational power for this type of analysis is becoming more and more sophisticated, resulting in characterisation and validation of new protein changes in response to chronic alcohol consumption. Furthermore, proteomic experiments are now able to detect thousands of proteins in a single run. In nonhuman primates an experimental 17-plasma protein panel (combining plasma cytokines, growth factors, and other proteins) correctly classified abusive drinking with $100 \%$ sensitivity and differentiated any level of drinking from alcohol abstinence with $88 \%$ accuracy [61].

Exemplary human serum proteins that can serve as novel biomarker candidates or components of biomarker panels to discern subjects with excessive alcohol use or confirm abstinence include AT-rich interactive domain-containing protein 4B, phosphatidylcholine-sterol acyltransferase, hepatocyte growth factor-like protein, ADP-ribosylation factor [62], serum amyloid A4, clusterin, fibronectin [61], $\alpha 2-H S$ glycoprotein, apolipoprotein A-I, glutathione peroxidase 3, heparin cofactor II, pigment epithelial-derived factor [63], a fragment of $\alpha$ fibrinogen, isoform 1 [64], gelsolin, selenoprotein $P$, serotransferrin, tetranectin, and haemopexin [65].

\section{Concluding remarks}

Because of the many limitations and weaknesses of currently used biomarkers of alcohol consumption, none of them has become widely accepted, and the search for ideal (i.e. more sensitive and specific) biomarkers continues. While it may be tempting to think of biomarkers as single molecules, a growing body of evidence indicates that panels of biomolecules in combination may function best in terms of sensitivity and specificity. 
Further investigations should elucidate important pathophysiological bases of alcohol drinking behaviour and ethanol-induced organ damage and ultimately lead to better forms of prevention and therapy. Importantly, future alcohol biomarkers need to be able to differentiate between a variety of drinking behaviours occurring in real clinical practice (abstinence vs. light drinking vs. heavy drinking), as opposed to just differentiating between nondrinking and drinking. Furthermore, they should also enable assessment of both average intake and drinking patterns (e.g. binge drinking).

Another challenge will be to translate often sophisticated and expensive analytical techniques for determining the chosen compounds in easily available fluids and tissue into cost-effective and straightforward diagnostic tools that can be used in routine clinical practice.

\section{Conflict of interest}

The authors declare no conflict of interest.

\section{References}

1. Parry CD, Patra J, Rehm J. Alcohol consumption and non-communicable diseases: epidemiology and policy implications. Addiction 2011; 106: 1718-24.

2. Greenfield TK, Ye Y, Kerr W, et al. Externalities from alcohol consumption in the 2005 US National Alcohol Survey: implications for policy. Int J Environ Res Public Health 2009; 6: 3205-24.

3. Gache P, Michaud P, Landry U, et al. The Alcohol Use Disorders Identification Test (AUDIT) as a screening tool for excessive drinking in primary care: reliability and validity of a French version. Alcohol Clin Exp Res 2005; 29: 2001-7.

4. Bradley KA, DeBenedetti AF, Volk RJ, et al. AUDIT-C as a brief screen for alcohol misuse in primary care. Alcohol Clin Exp Res 2007; 31: 1208-17.

5. Mackenzie D, Langa A, Brown TM. Identifying hazardous or harmful alcohol use in medical admissions: a comparison of audit, cage and brief mast. Alcohol Alcohol 1996; 31: 591-9.

6. Biomarkers Working Group. Biomarkers and surrogate endpoints: preferred definitions and conceptual framework. Clin Pharmacol Ther 2001; 69: 89-95.

7. Peterson K. Biomarkers for alcohol use and abuse - a summary. Alcohol Res Health 2004-2005; 28: 30-7.

8. Waszkiewicz N, Konarzewska B, Waszkiewicz M, et al. Biomarkers of alcohol abuse. Part I. Traditional biomarkers and their interpretation. Psychiatr Pol 2010; 44: 127-36.

9. Waszkiewicz N, Szajda SD, Kępka A, et al. Glycoconjugates in the detection of alcohol abuse. Biochem Soc Trans 2011; 39: 365-9.

10. Waszkiewicz N, Popławska R, Konarzewska B, et al. Biomarkers of alcohol abuse. Part II. New biomarkers and their interpretation. Psychiatr Pol 2010; 44: 137-46.

11. Wurst FM, Skipper GE, Weinmann W. Ethyl glucuronide - the direct ethanol metabolite on the threshold from science to routine use. Addiction 2003; 98: 51-61.
12. Morini L, Politi L, Zucchella A, Polettini A. Ethyl glucuronide and ethyl sulphate determination in serum by liquid chromatography-electrospray tandem mass spectrometry. Clin Chim Acta 2007; 376: 213-9.

13. Morini L, Colucci M, Ruberto MG, et al. Determination of ethyl glucuronide in nails by liquid chromatography tandem mass spectrometry as a potential new biomarker for chronic alcohol abuse and binge drinking behavior. Anal Bioanal Chem 2012; 402: 1865-70.

14. Høiseth G, Morini L, Polettini A, et al. Ethyl glucuronide in hair compared with traditional alcohol biomarkers - a pilot study of heavy drinkers referred to an alcohol detoxification unit. Alcohol Clin Exp Res 2009; 33: 812-6.

15. Kharbouche H, Faouzi M, Sanchez N, et al. Diagnostic performance of ethyl glucuronide in hair for the investigation of alcohol drinking behavior: a comparison with traditional biomarkers. Int J Legal Med 2012; 126: 243-50.

16. Reisfield GM, Goldberger BA, Crews BO, et al. Ethyl glucuronide, ethyl sulfate, and ethanol in urine after sustained exposure to an ethanol-based hand sanitizer. J Anal Toxicol 2011; 35: 85-91.

17. Thierauf A, Wohlfarth A, Auwärter V, et al. Urine tested positive for ethyl glucuronide and ethyl sulfate after the consumption of yeast and sugar. Forensic Sci Int 2010; 202: 45-7.

18. Yegles M, Labarthe A, Auwarter V, et al. Comparison of ethyl glucuronide and fatty acid ethyl ester concentrations in hair of alcoholics, social drinkers and teetotallers. Forensic Sci Int 2004; 145: 167-73.

19. Thiele GM, Klassen LW, Tuma DJ. Formation and immunological properties of aldehyde-derived protein adducts following alcohol consumption. Methods Mol Biol 2008; 447: 235-57.

20. De Benedetto GE, Fanigliulo M. A new CE-ESI-MS method for the detection of stable hemoglobin acetaldehyde adducts, potential biomarkers of alcohol abuse. Electrophoresis 2009; 30: 1798-807.

21. Romanazzi V, Schilirò T, Carraro E, et al. Immune response to acetaldehyde-human serum albumin adduct among healthy subjects related to alcohol intake. Environ Toxicol Pharmacol 2013; 36: 378-83.

22. Hietala J, Koivisto H, Latvala J, et al. IgAs against acetaldehyde-modified red cell protein as a marker of ethanol consumption in male alcoholic subjects, moderate drinkers, and abstainers. Alcohol Clin Exp Res 2006; 30: 1693-8.

23. Waszkiewicz N, Szajda SD, Jankowska A. The effect of acute ethanol intoxication on salivary proteins of innate and adaptive immunity. Alcohol Clin Exp Res 2008; 32: 652-6.

24. Wurst FM, Alexson S, Wolfersdorf M, et al. Concentration of fatty acid ethyl esters in hair of alcoholics: comparison to other biological state markers and self reported-ethanol intake. Alcohol Alcohol 2004; 39: 33-8.

25. Auwarter V, Sporkert F, Hartwig S, et al. Fatty acid ethyl esters in hair as markers of alcohol consumption. Segmental hair analysis of alcoholics, social drinkers, and teetotalers. Clin Chem 2001; 47: 2114-23.

26. Refaai MA, Nguyen PN, Steffensen TS, et al. Liver and adipose tissue fatty acid ethyl esters obtained at autopsy are postmortem markers for premortem ethanol intake. Clin Chem 2002; 48: 77-83. 
27. Pragst F, Balikova MA. State of the art in hair analysis for detection of drug and alcohol abuse. Clin Chim Acta 2006; 370: 17-49.

28. Pragst $F$, Yegles $M$. Determination of fatty acid ethyl esters (FAEE) and ethyl glucuronide (EtG) in hair: a promising way for retrospective detection of alcohol abuse during pregnancy? Ther Drug Monit 2008; 30: 255-63.

29. Hartwig S, Auwarter V, Pragst F. Effect of hair care and hair cosmetics on the concentrations of fatty acid ethyl esters in hair as markers of chronically elevated alcohol consumption. Forensic Sci Int 2003; 131: 90-7.

30. Himes SK, Dukes KA, Tripp T, et al. Prenatal Alcohol in SIDS and Stillbirth (PASS) Network. Clinical sensitivity and specificity of meconium fatty acid ethyl ester, ethyl glucuronide, and ethy sulfate for detecting maternal drinking during pregnancy. Clin Chem 2015; 61: 523-32.

31. Cabarcos P, Tabernero MJ, Otero JL, et al. Quantification of fatty acid ethyl esters (FAEE) and ethyl glucuronide (EtG) in meconium for detection of alcohol abuse during pregnancy: correlation study between both biomarkers. J Pharm Biomed Anal 2014; 100: 74-8.

32. Aradottir S, Moller K, Alling C. Phosphatidylethanol formation and degradation in human and rat blood. Alcohol Alcohol 2004; 39: 8-13.

33. Wurst FM, Thon N, Aradottir S, et al. Phosphatidylethanol: normalization during detoxification, gender aspects and correlation with other biomarkers and self-reports. Addict Biol 2010 15: 88-95.

34. Aradottir S, Asanovska G, Gjerss S, et al. Phosphatidylethanol (PEth) concentrations in blood are correlated to reported alcohol intake in alcohol-dependent patients. Alcohol Alcohol 2006; 41: 431-7.

35. Wurst FM, Thon N, Weinmann W, et al. Characterization of sialic acid index of plasma apolipoprotein $\mathrm{J}$ and phosphatidylethanol during alcohol detoxification - a pilot study. Alcoho Clin Exp Res 2012; 36: 251-7.

36. Javors MA, Johnson BA. Current status of carbohydrate deficient transferrin, total serum sialic acid, sialic acid index of apolipoprotein J and serum beta-hexosaminidase as markers for alcohol consumption. Addiction 2003; 98: 45-50.

37. Hannuksela ML, Liisanantti MK, Nissinen AE, et al. Biochemical markers of alcoholism. Clin Chem Lab Med 2007; 45: 953-61.

38. Waszkiewicz N, Szajda SD, Jankowska A, et al. The effect of the binge drinking session on the activity of salivary, serum and urinary beta-hexosaminidase: preliminary data. Alcohol Alcohol 2009; 43: 446-50.

39. Gong M, Garige M, Hirsch K, Lakshman MR. Liver Galbeta1, 4GIcNAc alpha2,6-sialyltransferase is down-regulated in human alcoholics: possible cause for the appearance of asialoconjugates. Metabolism 2007; 56: 1241-7.

40. Ghosh P, Hale EA, Lakshman MR. Plasma sialic-acid index of apolipoprotein J (SIJ): a new alcohol intake marker. Alcoho 2001; 25: 173-9.

41. Chrostek L, Cylwik B, Krawiec A, et al. Relationship between serum sialic acid and sialylated glycoproteins in alcoholics. Alcohol Alcohol 2007; 42: 588-92.

42. Cylwik B, Krawiec A, Chrostek L, et al. The effect of chronic alcohol drinking on the total concentration of sialic acid and lipid-bound sialic acid. Pol Merkur Lekarski 2009; 27: 101-4.
43. Sillanaukee P, Ponnio M, Jaaskelainen IP. Occurrence of sialic acids in healthy humans and different disorders. Eur J Clin Invest 1999; 29: 413-25.

44. Chrostek L, Cylwik B, Korcz W, et al. Serum free sialic acid as a marker of alcohol abuse. Alcohol Clin Exp Res 2007; 31: 9961001.

45. Beck O, Helander A. 5-hydroxytryptophol as a marker for recent alcohol intake. Addiction 2003; 98: 63-72.

46. Høiseth G, Bernard JP, Stephanson N, et al. Comparison between the urinary alcohol markers EtG, EtS, and GTOL/5-HIAA in a controlled drinking experiment. Alcohol Alcohol 2008; 43 : 187-91.

47. Hipólito L, Sánchez-Catalán MJ, Martí-Prats L, et al. Revisiting the controversial role of salsolinol in the neurobiological effects of ethanol: old and new vistas. Neurosci Biobehav Rev 2012; 36: 362-78.

48. Musshoff F. Chromatographic methods for the determination of markers of chronic and acute alcohol consumption. J Chromatogr B Analyt Technol Biomed Life Sci 2002; 781: 457-80.

49. Haber $\mathrm{H}$, Jahn $\mathrm{H}$, Ehrenreich $\mathrm{H}$, et al. Assay of salsolinol in peripheral blood mononuclear cells of alcoholics and healthy subjects by gas chromatography-mass spectrometry. Addict Biol 2002; 7: 403-7.

50. Lee J, Ramchandani VA, Hamazaki K, et al. A critical evaluation of influence of ethanol and diet on salsolinol enantiomers in humans and rats. Alcohol Clin Exp Res 2010; 34: 242-50.

51. Roine RP. Effects of moderate drinking and alcohol abstinence on urinary dolichol levels. Alcohol 1988; 5: 229-31.

52. Roine R, Nykänen I, Salaspuro M. Effect of alcohol on urinary and blood dolichols. Biochem Cell Biol 1992; 70: 404-7.

53. Achur RN, Freeman WM, Vrana KE. Circulating cytokines as biomarkers of alcohol abuse and alcoholism. J Neuroimmune Pharmacol 2010; 5: 83-91.

54. Afshar M, Richards S, Mann D, et al. Acute immunomodulatory effects of binge alcohol ingestion. Alcohol 2015; 49: 57-64.

55. Almeida J, Polvorosa MA, Gonzalez-Quintela A, et al. Decreased peripheral blood CD4+/CD25+ regulatory T cells in patients with alcoholic hepatitis. Alcohol Clin Exp Res 2013; 37: 1361-9.

56. Joosten MM, van Erk MJ, Pellis L, et al. Moderate alcohol consumption alters both leucocyte gene expression profiles and circulating proteins related to immune response and lipid metabolism in men. Br J Nutr 2012; 108: 620-7.

57. Gonzalez-Quintela A, Campos J, Loidi L, et al. Serum TNF-alpha levels in relation to alcohol consumption and common TNF gene polymorphisms. Alcohol 2008; 42: 513-8.

58. Laso FJ, Vaquero JM, Almeida J, et al. Production of inflammatory cytokines by peripheral blood monocytes in chronic alcoholism: relationship with ethanol intake and liver disease. Cytometry B Clin Cytom 2007; 72: 408-15.

59. Mischak H, Allmaier G, Apweiler R, et al. Recommendations for biomarker identification and qualification in clinical proteomics. Sci Transl Med 2010; 2: 46ps42.

60. Gorini G, Harris RA, Mayfield RD. Proteomic approaches and identification of novel therapeutic targets for alcoholism. Neuropsychopharmacology 2014; 39: 104-30.

61. Freeman WM, Vanguilder HD, Guidone E, et al. Plasma proteomic alterations in non-human primates and humans after chronic alcohol self-administration. Int J Neuropsychopharmacol 2011; 14: 899-911. 
62. Liangpunsakul S, Lai X, Ross RA, et al. Novel serum biomarkers for detection of excessive alcohol use. Alcohol Clin Exp Res 2015; 39: 556-65.

63. Sogawa K, Kodera Y, Satoh M, et al. Increased serum levels of pigment epithelium-derived factor by excessive alcohol consumption-detection and identification by a three-step serum proteome analysis. Alcohol Clin Exp Res 2011; 35: 211-7.

64. Liangpunsakul S, Lai X, Ringham HN, et al. Serum proteomic profiles in subjects with heavy alcohol abuse. J Proteomics Bioinform 2009; 2: 236-43.

65. Sogawa K, Satoh M, Kodera Y, et al. A search for novel markers of alcohol abuse using magnetic beads and MALDI-TOF/TOF mass spectrometry. Proteomics Clin Appl 2009; 3: 821-8.

66. Spiegel DR, Dhadwal N, Gill F. "I'm sober, Doctor, really": best biomarkers for underreported alcohol use. Curr Psychiatry 2008; 7: 15-27.

67. Substance Abuse and Mental Health Services Administration (SAMSHA) Advisory. The role of biomarkers in the treatment of alcohol use disorders, 2012 Revision. Available on-line: [http:// store.samhsa.gov/shin/content/SMA12-4686/SMA12-4686. pdf].

68. Pietrzak A, Jastrzębska I, Chodorowska G, et al. Psoriasis and unreported excessive alcohol intake - a simple screening approach. J Eur Acad Dermatol Venereol 2011; 25: 1261-8.

Received: 23.06.2015

Accepted: 24.12 .2015 